\title{
Self-assembled domain structures: From micro- to nanoscale
}

\author{
Vladimir Shur*, Andrey Akhmatkhanov, Alexey Lobov and Anton Turygin \\ Ferroelectric Laboratory, Institute of Natural Sciences \\ Ural Federal University, 51 Lenin Ave \\ Ekaterinburg 620000, Russia \\ *vladimir.shur@urfu.ru
}

Received 9 April 2015; Revised 8 May 2015; Accepted 11 May 2015; Published 22 June 2015

\begin{abstract}
The recent achievements in studying the self-assembled evolution of micro- and nanoscale domain structures in uniaxial single crystalline ferroelectrics lithium niobate and lithium tantalate have been reviewed. The results obtained by visualization of static domain patterns and kinetics of the domain structure by different methods from common optical microscopy to more sophisticated scanning probe microscopy, scanning electron microscopy and confocal Raman microscopy, have been discussed. The kinetic approach based on various nucleation processes similar to the first-order phase transition was used for explanation of the domain structure evolution scenarios. The main mechanisms of self-assembling for nonequilibrium switching conditions caused by screening ineffectiveness including correlated nucleation, domain growth anisotropy, and domain-domain interaction have been considered. The formation of variety of self-assembled domain patterns such as fractal-type, finger and web structures, broad domain boundaries, and dendrites have been revealed at each of all five stages of domain structure evolution during polarization reversal. The possible applications of self-assembling for micro- and nanodomain engineering were reviewed briefly. The review covers mostly the results published by our research group.
\end{abstract}

Keywords: Self-assembling; nanodomain structures; nanodomain engineering; lithium niobate; lithium tantalate.

\section{Introduction}

The production of the periodic domain structure in commercially available ferroelectric crystals is used for improvement of the properties important for application (domain engineering). ${ }^{1-3}$ The spatial modulation of the electro-optic, photorefractive, piezoelectric, and nonlinear optical properties allows one to develop the devices with upgraded performance. $^{3-5}$ The particular interest is related to creation of the self-similar and quasi-periodic domain structures with high wall concentration. The domain wall engineering is important for improvement of the piezoelectric and dielectric properties. ${ }^{6-9}$ The recent achievements in study of the domain structure evolution are conditioned by appearance of the domain visualization methods with high spatial resolution. It gave rise to the domain nanotechnology (nanodomain engineering) which can be used to manufacture the domain structures with nanometer precision ${ }^{10-13}$ and creation of the sub-micron-pitch domain gratings. ${ }^{14-17}$ The domain structure evolution in nanoscale is associated with self-assembled domain growth, domain shape instabilities, and formation of the self-similar domain patterns. ${ }^{3,18}$ The self-organized domain structures were recently observed by other scientific groups during pulsed laser irradiation. ${ }^{19,20}$

The crystals of lithium niobate $\mathrm{LiNbO}_{3}(\mathrm{LN})$ and lithium tantalate $\mathrm{LiTaO}_{3}$ (LT) family have been selected both as the most important for application nonlinear optical materials due to their large electro-optical and nonlinear optical coefficients and as the model ferroelectrics with simple domain structure with $180^{\circ}$ walls only and possibility to vary the bulk screening rate in wide range by temperature change. ${ }^{16}$ The composition of the studied representatives of LN and LT family changed from congruent to stoichiometric and included both undoped and doped by $\mathrm{MgO} .^{21-24}$

Nowadays LN and LT are the most widely used objects of the domain engineering. Application of electric field through lithographically defined electrodes for domain patterning is one of the most promising methods. However, high coercive electric fields and the strong effect of domain widening out of the area covered by electrode impose limitations on the period of short-pitch domain patterns. Thus, up to now the domain patterns with periods below one micron have not been fabricated.

\section{Experimental Study of Polarization Reversal and Domain Kinetics}

The micro- and nanodomain engineering has become recently one of the most important areas of ferroelectric science and technology. The main target of the domain engineering is the manufacturing of the stable tailored domain patterns in commercially available ferroelectrics in order to improve their applied characteristics. ${ }^{1,25}$ The periodic domain structures open the wide possibilities in the development of devices with upgraded performance owing to introduced

This is an Open Access article published by World Scientific Publishing Company. It is distributed under the terms of the Creative Commons Attribution 3.0 (CC-BY) License. Further distribution of this work is permitted, provided the original work is properly cited. 
spatial modulation of the electro-optic, photorefractive, piezoelectric, and nonlinear optical properties. ${ }^{26-29}$ The modern achievements in investigation of the ferroelectric domain structures and their evolution invoked by recent appearance of the domain visualization methods with high spatial resolution gave rise to the new developing stage of the domain engineering - domain nanotechnology (nanodomain engineering). ${ }^{2,3,16}$ The purposes of nanodomain engineering are manufacturing of the periodical domain structures with nanoscale precision and creation of the sub-micron-pitch domain gratings. The deeper research of the domain structure evolution in nanoscale should be associated with selfassembled domain growth, domain shape instabilities, and formation of the self-similar (fractal) domain patterns.

Z-cut wafers of various representatives of LN and LT family have been examined. For the in situ investigation of the domain kinetics in a uniform electric field the 1-mmdiameter circular transparent electrodes of two types were used: (1) liquid electrolyte (water solution of $\mathrm{LiCl}$ ) in a special fixture and (2) $\mathrm{In}_{2} \mathrm{O}_{3}: \mathrm{Sn}$ (ITO) films deposited by magnetron sputtering. The direct observations of the domain evolution were carried out using a polarizing microscope with simultaneous video recording and subsequent processing of the image set.

The number of different experimental techniques ranging from common optical microscopy to more sophisticated piezoelectric force microscopy (PFM), scanning electron microscopy (SEM), and confocal Raman microscopy (CRM) have been used for the investigation of the static domain patterns and the kinetics of the domain structure in crystals of LN and LT family. A review of modern experimental methods has been published recently. ${ }^{30,31}$

The known methods used for visualization of the static domain patterns ${ }^{30,31}$ can be divided into two groups. The methods of the first group require the preliminary revealing of the domain pattern by selective chemical etching, while the second group does not require any treatment. It is clear that any preliminary treatment is undesirable because it can significantly affect the investigated domain pattern. ${ }^{32}$

\subsection{Selective chemical etching}

It is easy to obtain the surface relief corresponding to the domain pattern at the polar surface in LN and LT since the etching rates for $Z^{-}$face are hundreds times faster than for $Z^{+}$one for etching by HF acid. ${ }^{33}$ The revealed relief can be visualized by optical microscopy, SEM and AFM..$^{30,34,35}$ The visualization by SEM provides the highest available spatial resolution which is below $2 \mathrm{~nm}$, and ability to obtain the surface localization in depth below $100 \mathrm{~nm}$.

\subsection{Visualization without any treatment}

The large linear electro-optic effect in LN and LT makes possible optical visualization of domains with neutral and charged walls using polarized and phase contrast modes. The method allows one to realize in situ visualization of the domain kinetics with high temporal resolution limited by the frame rate of CCD camera only.

PFM allows one to visualize the domain patterns at the polar surfaces with nanoscale spatial resolution. The local values of amplitude and phase of the piezoelectric response are recorded during scanning along the surface. The phase signal allows to reveal the domain contrast, whereas the amplitude signal can be used for visualization of the domain walls with the resolution ranged from 10 to $30 \mathrm{~nm}^{34-39}$

CRM is able to visualize the domain structure in the bulk with the spatial resolution below the diffraction limit. ${ }^{40-42}$ The set of two-dimensional (2D) domain images obtained in the bulk using layer by layer scanning at various depths can be used for extracting the information about the time evolution of the domain structure at the polar surface. ${ }^{41,42}$ The earlier moments of the domain evolution at the sample polar surface can be restored from layers scanned deeper in the bulk. ${ }^{31}$

\section{Kinetic Approach to Domain Structure Evolution}

The domain structure evolution can be considered as a manifestation of the various nucleation processes similar to the firstorder phase transition. ${ }^{43,44}$ Within this kinetic approach the neighboring domains are similar to the volumes of different phases divided by interfaces (domain walls). The domain structure evolution can be considered as a result of thermally activated generation of one-, two-, and three-dimensional nuclei (1D-, 2D-, and 3D-nucleation) with preferred orientation of the spontaneous polarization.

The domain wall motion is a result of step generation at the wall (2D-nucleation) and step growth along the wall (1D-nucleation). The nucleation probability is determined by the local value of the electric field averaged over the nuclei volume (the driving force). ${ }^{1,4,45}$

The shape of isolated domain is defined by position of the nucleation sites at the domain walls. The usual hexagon domain shape in $\mathrm{LN}$ with walls oriented along $Y$-directions is caused by the determined nucleation at three nonadjacent domain only and step propagation along three allowed Y-directions (Fig. 1). ${ }^{4}$

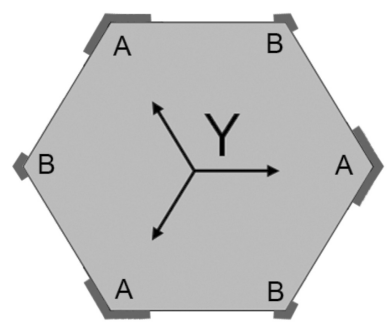

Fig. 1. Determined nucleation in LN crystal. 


\section{Main Mechanisms of Self-Assembling}

\subsection{Screening ineffectiveness}

The nucleation probability for domain growth is governed by local value of the electric field averaged over the nucleus size ("local field" $\left.\left.E_{\mathrm{loc}}\right)\right)^{4,45}$ It is clear that $E_{\mathrm{loc}}$ is spatially inhomogeneous and changes essentially during polarization reversal. $E_{\text {loc }}$ includes several components: (1) the external field $\left(E_{\mathrm{ex}}\right)$ produced by applied voltage, $(2)$ the residual depolarization field $\left(E_{\mathrm{rd}}\right)$ produced by bound charges and external screening charges, which depends on the domain shape and sizes, (3) the bulk screening field $\left(E_{b}\right)$ governed by bulk screening processes. ${ }^{46,47}$

$$
E_{\mathrm{loc}}(r, t)=E_{\mathrm{ex}}(r)+E_{\mathrm{rd}}(r, t)+E_{b}(r, t) .
$$

Our approach to the field induced domain kinetics is based on the consistent accounting of the bulk screening effects. The residual depolarization field $E_{\mathrm{rd}}$ remains in the bulk of the switched areas after completion of the external screening due to existence of the intrinsic or artificial dielectric surface layer (gap) in ferroelectric capacitor. ${ }^{45,48}$ In typical experimental conditions, $E_{\mathrm{rd}}$ value is close to threshold field $\left(E_{\mathrm{th}}\right)$ needed for beginning of the nucleation process.

The three groups of the bulk screening mechanisms can be considered: (1) redistribution of the bulk charges, ${ }^{45,48}$ (2) reorientation of the defect dipoles, ${ }^{49}$ (3) injection of the carriers from the electrode through the dielectric gap. ${ }^{50,51}$ All bulk screening mechanisms are slow with time constants range from milliseconds to days and months. The retardation of bulk screening can be characterized by screening ineffectiveness $(R)$ which represents the ratio between switching rate $\left(1 / t_{s}\right)$ and bulk screening rate $\left(1 / \tau_{\text {scr }}\right)^{4}$ :

$$
R=\tau_{\mathrm{scr}} / t_{s}
$$

The three ranges of the screening conditions can be distinguished: (1) $R \ll 1-$ complete screening, (2) $R \sim 1-$ incomplete screening, and (3) $R \gg 1$ - ineffective screening. It has been revealed experimentally and by computer simulation that the proposed ranges correspond to qualitatively different scenarios of the domain structure evolution. ${ }^{4}$

The complete screening $(R \ll 1)$ represents the quasiequilibrium switching conditions, when the bulk screening is fast enough to keep pace with the changes of $E_{\mathrm{rd}}$ and results in classical layer-by-layer domain wall motion. The incomplete screening $(R \sim 1)$ makes the switching conditions nonequilibrium resulting in deceleration of the classical wall motion and formation of the finger-type structure ("fingering"). 4,16 The impediment of the approaching walls results in existence of the residual micro- and nanodomains after ending of the switching process. The ineffective screening $(R \gg 1)$ makes the switching conditions highly nonequilibrium which leads to "discrete switching" by formation of the self-organized nanodomain structures, such as broad domain boundary (BDB) and web-like nanodomain ensembles. ${ }^{1,4}$

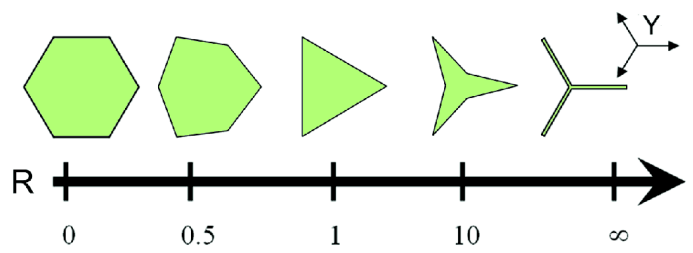

Fig. 2. Domain shapes obtained during computer simulation for various values of screening inefficiency.

We have investigated and verified by computer simulation the kinetic nature of the domain shape. It is clear that the observed wall orientation would be determined by the step concentration. A similar effect is observed for vicinal faces during crystal growth. For very low step concentration caused by much faster step growth as compared with the step generation the classical hexagons have to be observed. The simulation of the domain growth for wide range of $R$ values allows us to obtain the smooth variation of the domain shapes from equilateral hexagon to stars with three rays (Fig. 2).

\subsection{Correlated nucleation}

Formation of the self-assembled domain structures is driven by the correlated nucleation effect in the vicinity of the domain wall. ${ }^{45,52-55}$ The origin of the effect is the appearance of the local field maximum in front of the moving domain wall for ineffective screening conditions in the ferroelectric capacitor with surface dielectric gap. ${ }^{45,54-56}$ It has been shown by computer simulation that the pronounced maximum of the local electric field is situated at the boundary of the ferroelectric medium and the surface dielectric gap at the distance from the wall about the thickness of the dielectric gap L (Fig. 3). ${ }^{4}$

The correlated nucleation leads to formation of quasiregular structure of isolated micro- and nanodomain for ineffective screening conditions. It is known that appearance of the trail of partially screened residual depolarization field
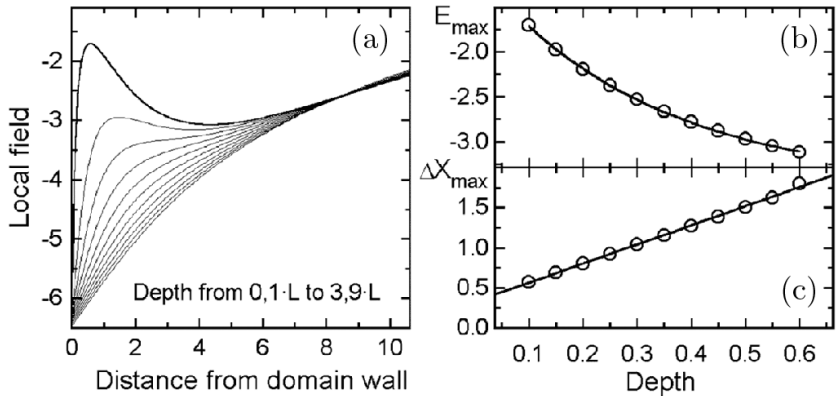

Fig. 3. (a) The calculated local field distribution near the stripe nonthrough domain at different depths from the surface dielectric layer. (b) Local field maximum $E_{\max }$ and (c) distance from the wall to the position of field maximum $x_{\max }$ versus the depth. The distance from the wall and the depth are divided by the thickness of the surface dielectric layer L. Reprinted with permission from Ref. 4. Copyright 2005, Wiley-VCH Verlag GmbH \& Co. KGaA. 
behind the moving domain wall decelerates and even terminates the classical wall motion by step generation and growth along the wall (step-by-step growth). In addition, the field produced by the trail increases the $3 \mathrm{D}$-nucleation probability at the certain distance in front of the moving wall which results in appearance of isolated nanodomains. The correlated nucleation effect leads to formation of the quasi-regular domain chains aligning along the domain walls.

\subsection{Growth anisotropy}

The experimentally observed anisotropy of the step growth along the wall can be attributed to anisotropy of the bulk conductivity. We assume that the hopping electronic type conductivity dominates in LN and LT crystals at RT is anisotropic along $Y$ polar axis. ${ }^{1}$ This assumption allows us to explain the anisotropic growth of elementary steps along the domain wall, which leads to formation of the plane walls. ${ }^{1}$ Moreover, the nonequivalence of $Y^{+}$and $Y^{-}$crystallographic directions of $Y$ polar axis leads to the growth of strictly oriented nanodomain chains and arrays.

\subsection{Domain-domain interaction}

The isolated domains interact with each other, resulting in feedback-type mechanisms that strongly affect the self-organized domain kinetics. The domain-domain interaction was thoroughly studied in LN by SPM. Application of field pulses to the SPM tip in contact with the surface led to formation of domain which can be detected in PFM mode. ${ }^{37,57}$ Application of the set of field pulses during scanning resulted in a formation of the domain chain. The uniform domain size is an indicator of the high LN quality. Generally, domain growth
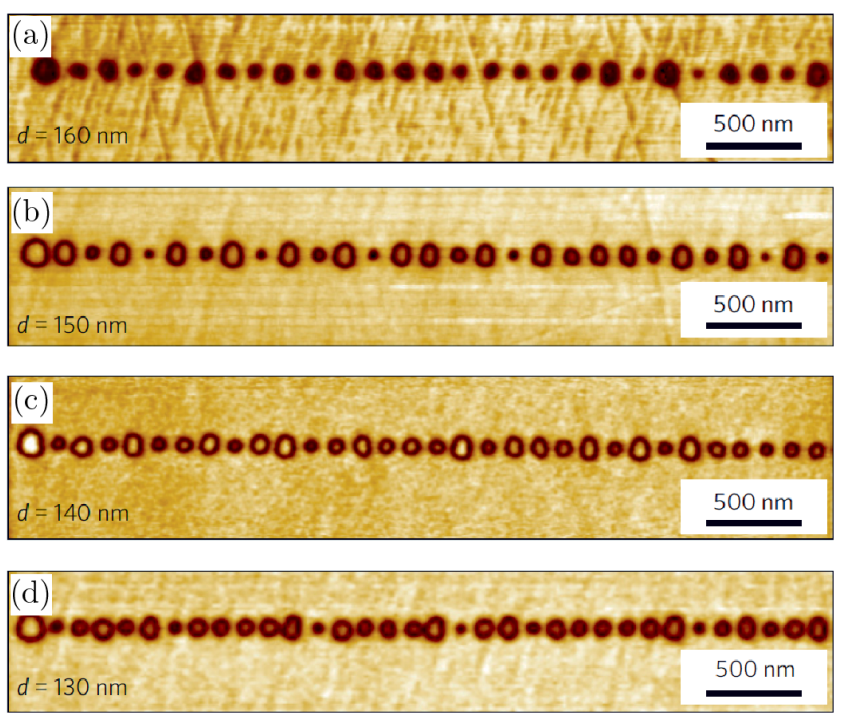

Fig. 4. Evolution of domain morphologies in a single chain as a function of domain-domain distance. $U_{\mathrm{sw}}=40 \mathrm{~V}$. Note (b) the clearly visible period doubling and $(\mathrm{c}, \mathrm{d})$ intermittent behaviors. ${ }^{52}$ follows kinetic laws with domain size proportional to the tip bias (above a certain threshold corresponding to domain nucleation) and approximately logarithmic with the pulse time. $^{58}$

The unusual effects are observed when the spacing between the points of bias field application is varied (Fig. 4). ${ }^{59}$ On decreasing the distance between the field application sites, the domain sizes become nonequal. The intermittency is observed, when only every second site of bias application contains the domain, and also nonequal domain sizes with double periodicity were observed. On further distance decrease, the period tripling and formation of aperiodic or long-periodic structures were observed. ${ }^{59}$

The simulation of the field distribution in the vicinity of the isolated domain has demonstrated the maximum of the switching field at certain distance from the domain wall. ${ }^{59}$

\section{How to Achieve Nonequilibrium Switching Conditions}

The completely ineffective screening of $E_{\mathrm{rd}}$ can be achieved by acceleration of the domain kinetics or by increasing of $E_{\mathrm{rd}}$ using various methods: (1) superfast switching, (2) creation of the artificial surface dielectric layer, (3) switching by pyroelectric field appeared during cooling after pulse heating.

\subsection{Superfast spontaneous backswitching}

The acceleration of the switching by application of the strong electric field is the simplest way to obtain nonequilibrium conditions, but its application in congruent LN (CLN) and congruent LT (CLT) is hampered due to extremely high threshold fields. That is the reason to use backswitching after rapid electric field switch-off. ${ }^{27,60,61}$ The backswitching under the action of the internal charges allows one to avoid the electrical breakdown. The backswitched poling has been applied successfully for the first creation of the nanoscale domain structure in CLN. ${ }^{26}$

\subsection{Creation of the artificial dielectric surface layer}

Increasing of $E_{\mathrm{rd}}$ can be achieved by creation of the artificial surface dielectric layer by deposition of uniform dielectric film or by modification of the surface layer. The spinning of photoresist and deposition of the dielectric film, as well as surface modification by proton exchange ${ }^{54,55,62,63}$ and ion irradiation $^{64}$ were used. The tailored thickness of the dielectric gap allows one to control the period of the self-organized nanodomain structures.

\subsection{Switching under the action of the pyroelectric field}

The almost complete suppression of the external screening can be realized for switching without external circuit under 
the action of the pyroelectric field during cooling after pulse heating. ${ }^{65-67}$ The formation of the nanodomain structures has been obtained during cooling after heating by pulse infrared laser $(\lambda=10.6 \mu \mathrm{m}){ }^{68,69}$ The revealed rules of the oriented growth of nanodomain chains strictly along $Y$ crystallographic axis followed by reflection of interacting chains and branching allowed one to produce the stable self-assembled nanodomain structures with the depth up to hundreds of microns. ${ }^{66,67,70}$

\section{Five Stages of Domain Evolution during Polarization Reversal}

The five main stages of the domain structure evolution can be revealed during polarization reversal from the single domain state: (1) nucleation of new domains, (2) forward domain growth, (3) sideways domain growth, (4) domain coalescence, and (5) spontaneous backswitching (Fig. 5). ${ }^{1}$

Nucleation of new domains is the stage which is the most difficult for direct experimental study as it requires visualization of the appearance of isolated nanoscale needle-like domains (Fig. 5(a)). Moreover, it is still under discussion if the initial domain state is really single-domain or it contains irremovable nanodomains. ${ }^{45}$

Forward domain growth represents a relatively fast expansion of the needle-like domains through the sample in polar direction by rapid motion of the domain tip (Fig. 5(b)). The direct observation of this comparatively short stage

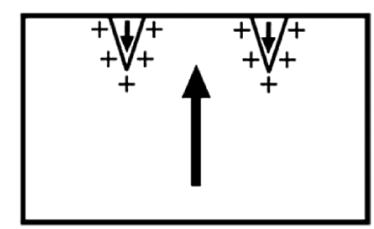

(a)

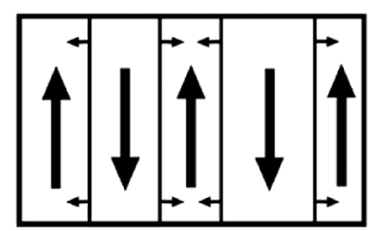

(c)

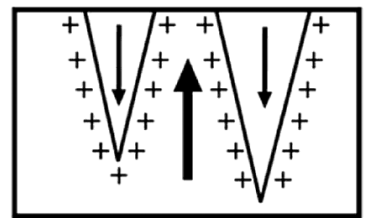

(b)

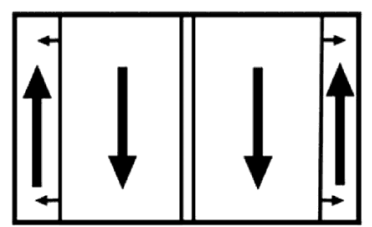

(d)

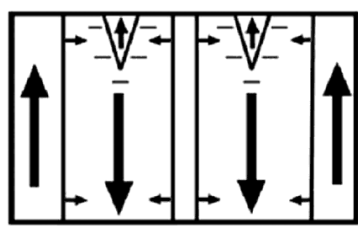

(e)

Fig. 5. The main stages of the domain structure evolution during polarization reversal from the single domain state. ${ }^{1}$ encounters experimental difficulties and is still poorly explored.

Sideways domain growth (spreading) stage is the best studied experimentally by in situ optical methods, which possess high temporal but insufficient spatial resolutions. At this stage the domain growth is achieved through the domain wall motion in the direction perpendicular to the polar one (Fig. 5(c)).

Coalescence of residual domains (merging) occurs when the polarization reversal is close to the completion (Fig. 5(d)). This stage is characterized by pronounced deceleration of the approaching domain walls due to electrostatic interaction. The incomplete merging leads to formation of the residual isolated nanodomains and net structures. ${ }^{54}$

Spontaneous backswitching (flip-back) after applied electric field switch-off represents partial restoration of the initial domain state by backward wall motion and by appearance of the domains with initial direction of the spontaneous polarization (Fig. $5(\mathrm{e}))^{26,60}$

According to above discussed mechanisms the formation of the self-assembled domain structures can be realized at any stage of the domain evolution during polarization reversal. Let us discuss the manifestations of self-assembling at different stages.

\section{Self-Assembling for Nucleation}

The formation of the dense nanodomain self-assembled structure was observed in CLN with polar surface modified by proton exchange (Fig. 6). ${ }^{54,55}$ The structure appeared between approaching walls of two large hexagonal domains during polarization reversal. Analysis of the set of CRM domain images obtained at different depths revealed the main stages of the structure formation. At the beginning, the oriented growth along $Y$-direction of the nanodomain chains connecting the approaching domains prevailed (Fig. 6(a)). Further, the chains of the first generation merged into domain rays (trunks). Finally, the branching (growth of the short domain chains) started from the primary trunks. The
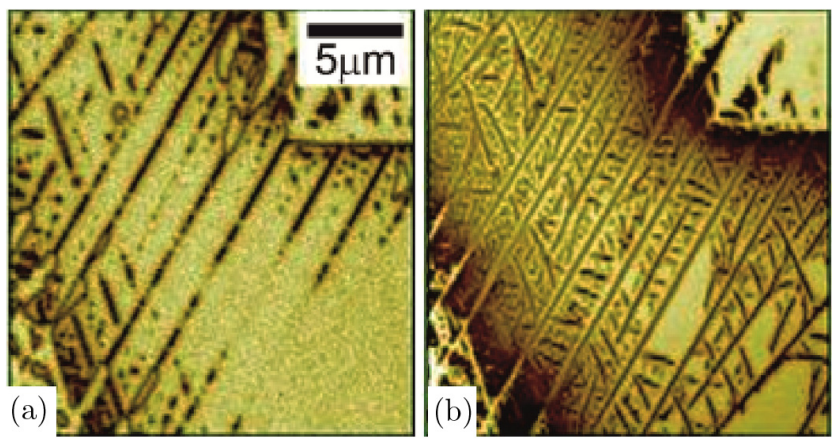

Fig. 6. Self-assembled nanodomain rays in CLN with surface layer modified by proton exchange. Images at different depths obtained at modified $\mathrm{Z}^{-}$surface and deeper: (a) $68 \mu \mathrm{m}$ and (b) at the surface. 
branching with subsequent merging resulted in formation of very dense patterns of nanodomain rays, which could be seen close to the modified surface (Fig. 6(b)).

\subsection{Broad domain boundary}

The formation of the correlated domain structure was visualized by CRM in CLN with the $1.7 \mu$ m-thick surface layer modified by proton exchange after partial switching $(E=$ $21.0 \mathrm{kV} / \mathrm{mm}){ }^{54,55}$ It was found that polarization switching in CLN with PE layer led to arising of the chains of needle-like domain in the vicinity of the domain walls.

The isolated domains grew in polar direction and finally merged with the initial domain. The region with a huge concentration of nonthrough domains enlarging by arising of new domain chains and slow domains growth was called BDB ${ }^{54}$ It was noticed that appeared regular domain structure was arranged according to the crystal symmetry. It looks like the revealed average domain size of BDB about $200 \mathrm{~nm}$ was limited by CRM spatial resolution. ${ }^{55}$ The depth of BDB (length of isolated needle-like nanodomains) extracted by analysis of the set of bulk CRM images ranged from 20 to $50 \mu \mathrm{m}$. It should be noted that the high-resolution PFM and SEM methods cannot be used for domain visualization at the polar surfaces modified by proton exchange. ${ }^{55}$

It was shown by optical microscopy that polarization reversal process started with appearance and growth of the isolated hexagonal domains, similarly to conventional switching. ${ }^{55}$ The intensive nucleation at the hexagon vertices led to lack of the domain shape stability for domain size above $20 \mu \mathrm{m}$ and to formation of the nanodomain structure in front of the moving wall (Fig. 7). The width of the stripe nanodomain area increased with domain growth. ${ }^{55}$

The similar BDB domain structure in CLN with photoresist layer was visualized by CRM and also by PFM with higher spatial resolution. ${ }^{71}$ It was shown that BDB represented the micron-size domains surrounded by the nanodomain chains (arrays) oriented along $Y$-directions. The diameter of the individual nanodomains ranged from 30 to $60 \mathrm{~nm}$. The average nanodomain period in arrays was about $90 \mathrm{~nm}$.

Formation of the nanoscale domain structure in the surface layer has been revealed in CLN crystals irradiated by

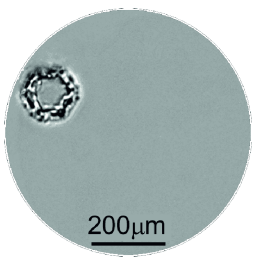

(a)

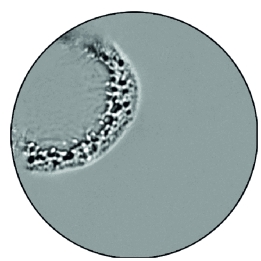

(b)

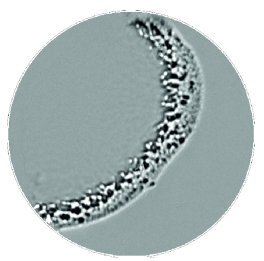

(c)
Fig. 7. In situ optical visualization of BDB formation in CLN with $1.7 \mu \mathrm{m}$-thick PE layer during polarization reversal. $E=21.5 \mathrm{kV} /$ $\mathrm{mm}$. Time from switching start: (a) $0.07 \mathrm{~s}$, (b) $0.34 \mathrm{~s}$ and (c) $0.82 \mathrm{~s}{ }^{48}$
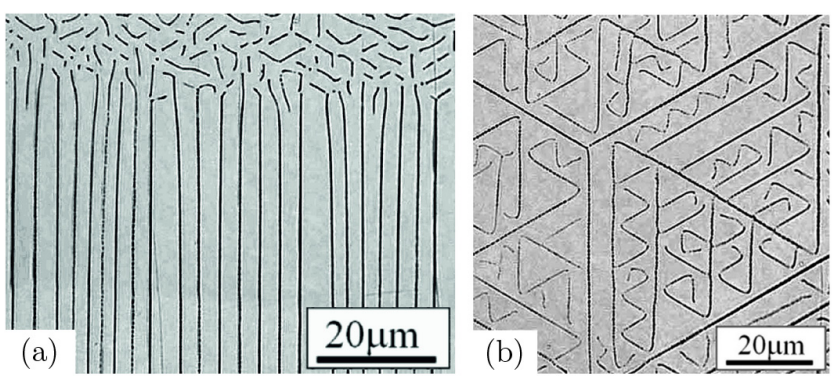

Fig. 8. (a) Quasi-periodic and (b) fractal-type domain structures in CLN after pulse laser irradiation.

pulse UV laser. ${ }^{65}$ The obtained dot\&dash, rays, and fractaltype self-assembled structures have been analyzed statistically. The optical observations clearly demonstrated that domain rays have grown strictly along $\mathrm{Y}^{+}$crystal directions (Fig. 8). It has been shown by high-resolution microscopy that the rays represent the chains of individual needle-like nanodomains. Computer simulation of the domain structure formation by growth and reflecting of the rays accompanied by their branching based on experimentally revealed rules was carried out. ${ }^{65}$ The simulated structures are in good agreement with the experimental ones. The fractal analysis of the domain clusters proved their self-similarity in wide size range.

\subsection{Dendrites}

The formation of the complicated self-organized dendrite-like domain structures was observed during polarization reversal at the elevated temperatures. Two stages of the dendrite growth have been separated: (1) "fingering" representing the

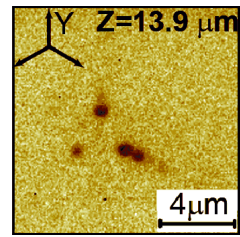

(a)

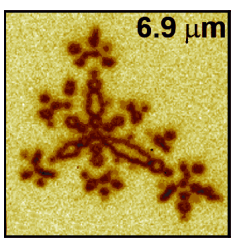

(d)

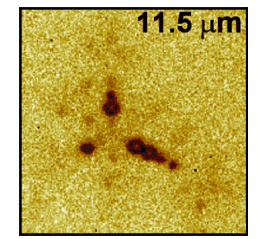

(b)

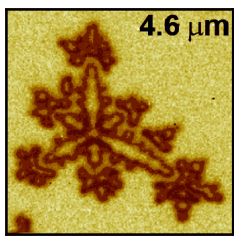

(e)

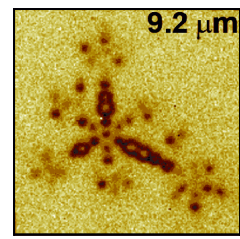

(c)

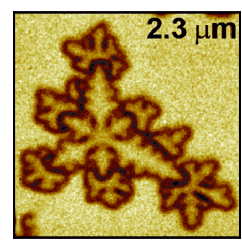

(f)
Fig. 9. The formation of the domain structures during application of two pulses extracted from the CRM images obtained at the different depths: (a)-(c) hexagonal domain star during the first pulse, (d)-(f) dendrite domain structure during the second pulse. Reprinted with permission from Ref. 73. Copyright 2012, AIP Publishing LLC. 
loss of the wall shape stability ${ }^{72}$ and (2) "branching" by formation of the new arrays starting from the initial one.

The formation of dendrite domain shapes has been studied in SLN crystals during polarization reversal at $T>230^{\circ} \mathrm{C}$ (Fig. 9). ${ }^{73}$ The independent domain growth after merging at the elevated temperatures caused by dominating of stochastic nucleation resulted in complicated domain shapes including dendrites. The domain shape was close to six-ray stars with vertices oriented along six $Y$-directions with the length of $Y^{+}$ rays essentially longer than of $Y^{-}$ones.

The main stages of the dendrite structure formation for application of the single pulse were revealed. (1) Appearance of a single isolated domain. (2) Appearance of three isolated domains at the equal distances from the central one in $\mathrm{Y}^{+}$directions suppressing its further growth. (3) Appearance of three isolated domains at the equal distances from the central domain in $Y$-directions. (4) Oriented growth of the domain chains with a sub-micron period in $\mathrm{Y}^{+}$ directions. (5) Formation of six-ray stars as a result of domain merging. (6) Formation of star-like structure as a result of ray broadening. ${ }^{73}$

\section{Self-Assembling for Forward Growth}

The self-assembled dense quasi-regular domain structures appeared in stoichiometric LT (SLT) crystals doped with MgO (MgOSLT) during polarization reversal in low field applied by solid-state electrodes. ${ }^{74-76}$ The domain structure was revealed by deep etching at $Z^{-}$polar surface (Fig. 10). It was shown that the average structure period $(1.2 \pm 0.1) \mu \mathrm{m}$ is independent on the applied external field. This fact confirms the self-organized nature of the obtained structures. The structure was attributed to formation and growth of the charged domain walls (CDW) situated close to $Z^{-}$polar surface. The CDW aperture measured by CRM was about $10 \mu \mathrm{m}$. The appearance of CDW leads also to significant increase of the low frequency dielectric permittivity as compared to the initial single domain state. ${ }^{74}$

The formation and field-induced evolution of self-organized CDW was investigated in CLN at room temperature using in situ methods. ${ }^{77}$ The optical-grade single-domain 0.2 -mm-thick wafers cut perpendicular to the polar axis with typical area $6 \times 6 \mathrm{~mm}^{2}$ were used. Transparent $\operatorname{In}_{2} \mathrm{O}_{3}: \mathrm{Sn}$ electrodes $\left(3 \mathrm{~mm}\right.$ diameter) were deposited on $\mathrm{Z}^{+}$and $Z^{-}$ surfaces. Rectangular voltage pulses with amplitude up to $5 \mathrm{kV}$ and duration ranging from 1 to $30 \mathrm{~s}$ were applied to the wafer at room temperature. In the experimental conditions used, the domains nucleated at the $\mathrm{Z}^{+}$surface, grew in the forward direction, and coalesced at $\mathrm{Z}^{+}$, but did not reach the $Z^{-}$surface, thus forming large isolated domains bounded by the CDW (Fig. 11). A strong dependence of CDW shape on the value and sign of the applied field was observed. ${ }^{77}$ The mechanism of CDW self-maintained propagation governed by self-consistent electrostatic interaction between the wall's steps and formation of the quasi-regular dented structure was proposed (Fig. 11(d)). ${ }^{77}$

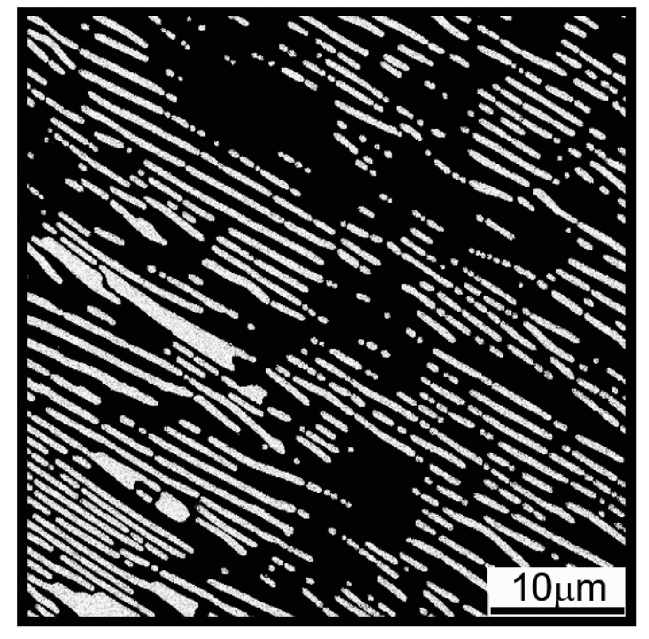

Fig. 10. Domain structure revealed by selective chemical etching and visualized by AFM in MgOSLT.

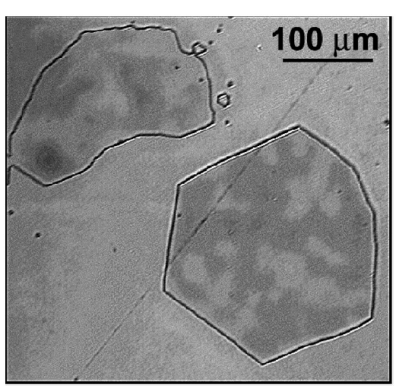

(a)

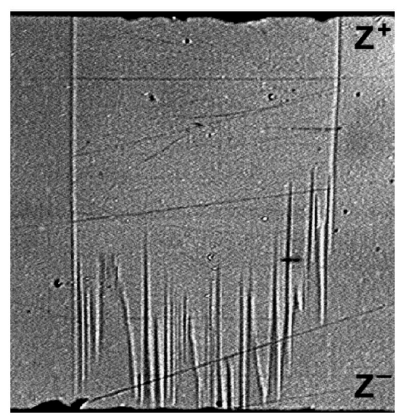

(c)

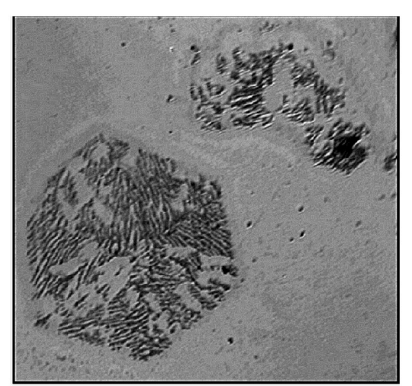

(b)

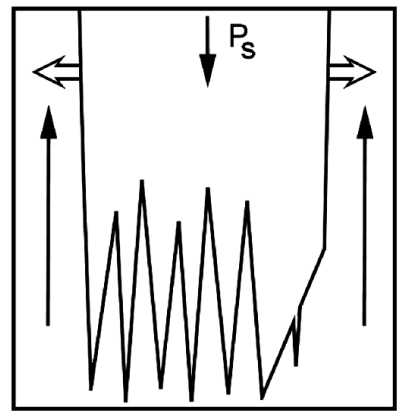

(d)
Fig. 11. CDW (a) $\mathrm{Z}^{+}$view, (b) $Z^{-}$view, (c) $Y$ view, and (d) scheme ( $Y$ view, white arrows - direction of boundary propagation), patterns revealed by etching and visualized by optical microscope; sample thickness $0.2 \mathrm{~mm}$. Reprinted with permission from Ref. 77 . Copyright 2000, AIP Publishing LLC.

\section{Self-Assembling for Sideways Wall Motion}

The classical step-by-step sideways motion of the planar domain wall can be completely suppressed by ineffective screening of $E_{\mathrm{rd}}$. In this case, the alternative mechanism of ongoing wall motion by formation and growth of protuberances takes place, which leads to lack of the domain wall 


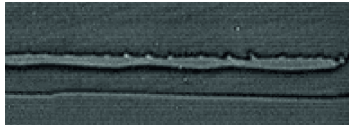

(a)

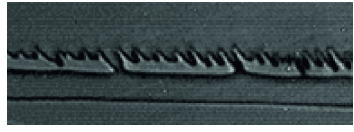

(b)

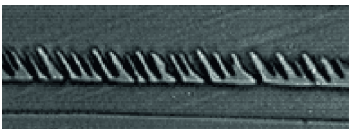

(c)

Fig. 12. Stages of the lack of the wall shape stability through formation of the finger structure in CLN with artificial dielectric layer. Optical observation of domains was revealed by etching. Reprinted with permission from Ref. 4. Copyright 2005, Wiley-VCH Verlag GmbH \& Co. KGaA.

shape stability and to formation of the self-assembled domain structure with sub-micron fingers (Fig. 12). ${ }^{4}$

The first protuberance induced by the local field inhomogeneity grew leading to formation of a single finger oriented along the $Y$-crystallographic direction (Fig. 12(a)). The correlated nucleation effect stimulates the self-assembled generation of the neighboring fingers and results in formation of the quasi-regular finger structure (Figs. 12(b) and 12(c)).

\section{Self-Assembling for Domain Merging}

It was shown that the deceleration of the motion of approaching parallel $Y$-oriented walls of hexagonal domains before merging in CLN-PE leads to formation of residual domains in the area where the merging occurred (Fig. 13). The formation of the chains of residual domains of lenticular shape was revealed by PFM. Pronounced maximum in the self-correlation function of the image of domains chain is the evidence of their correlated formation. Average structure period is about $20 \mu \mathrm{m}$, the domain width is about $1 \mu \mathrm{m}$ and the depth is about $40 \mu \mathrm{m}$ (Fig. 13).

\section{Self-Assembling for Backswitching}

Correlated nucleation has been obtained for the first time in CLN during backswitching after the abrupt external field switch-off (Fig. 14(a)). This effect has been attributed to the record-breaking value of spontaneous polarization in CLN, leading to extremely high value of the bulk screening field. After the external field switch-off, the local field changes the sign for incomplete screening. ${ }^{14,26}$

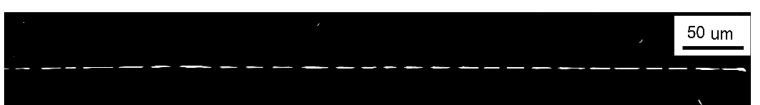

Fig. 13. Residual domains in CLN-PE appeared during domain merging. PFM visualization.
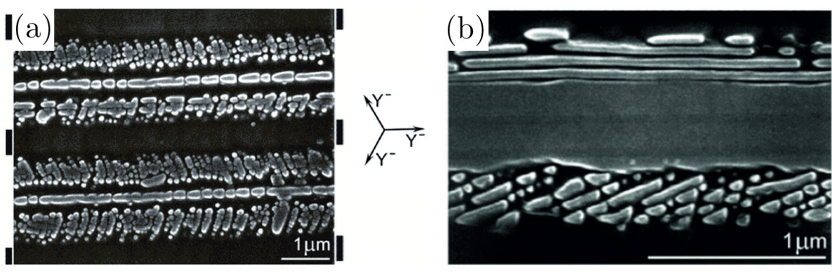

Fig. 14. Nanodomain arrays in periodically poled CLN oriented along $\mathrm{Y}^{+}$directions. $\mathrm{Z}^{+}$view. Domain patterns revealed by etching and visualized by SEM. Reprinted with permission from Ref. 14. Copyright 2000, AIP Publishing LLC.

It has been shown that the backward domain wall motion is achieved through enlarging of the self-assembled nanodomain structures (Fig. 14(a)). The nanodomain arrays has been revealed during backswitching in CLN in the vicinity of the stripe electrodes under artificial dielectric layer. ${ }^{4,14,26}$ The arrays were oriented mostly along $Y$ crystallographic directions and consisted of 30-100-nm-diameter needle-like domains (Fig. 14(b)). Similar quasi-regular nanodomain structures appeared in CLN with waveguides in the area covered by dielectric surface layer formed by proton exchange (Fig. 15). ${ }^{15}$

The alternative scenario of the backward wall motion is realized through formation and propagation of the quasiregular structure of fingers strictly oriented along $Y$-directions, which differ for different sides of the stripe domain (Fig. 16(b)). These finger structures grow towards each other, but the electrostatic interaction between approaching fingers prevents their merging. ${ }^{4}$ The same effect prevents merging of the neighboring fingers. As a result, the stable structure of residual domains forms (Fig. 16(b)).

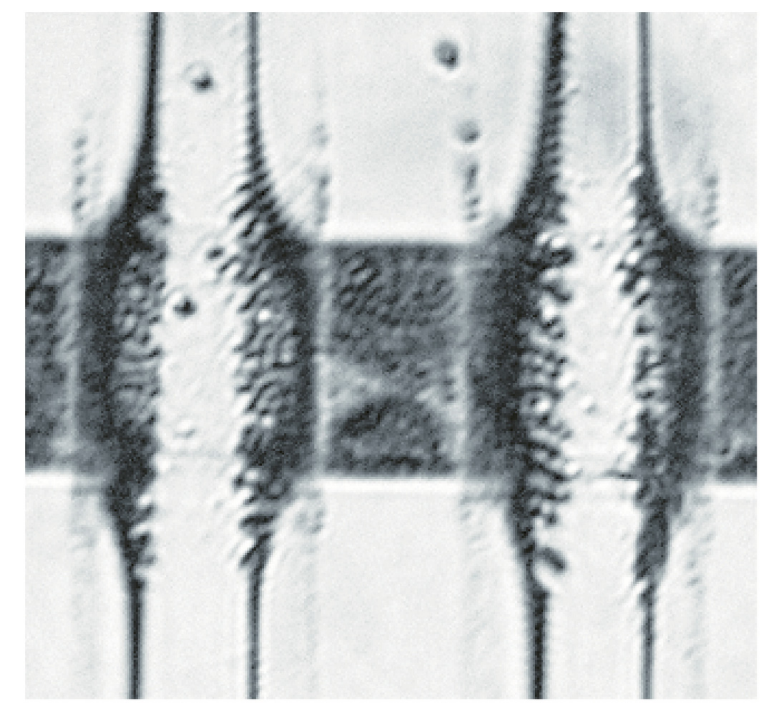

Fig. 15. Sub-micron scale domain arrays appeared during poling of CLN with proton exchanged waveguides visualized by optical microscope. 


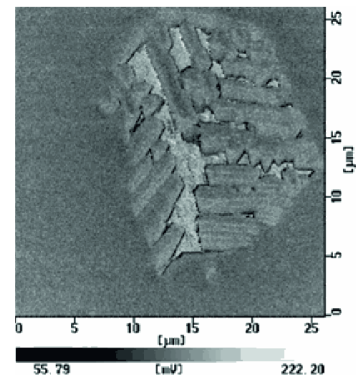

(a)

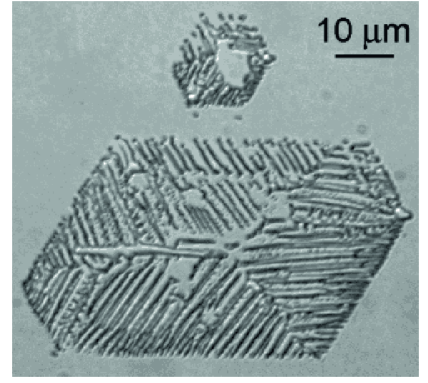

(b)
Fig. 16. (a) Backswitching in CLN. AFM image. (b) Hexagonal dendrite domain structure formed in $\mathrm{MgO}: \mathrm{LN}$ with artificial dielectric layer. Optical observation. The domains revealed by etching. Reprinted with permission from Ref. 4. Copyright 2005, Wiley-VCH Verlag GmbH \& Co. KGaA.

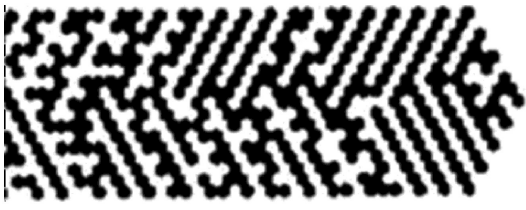

(a)

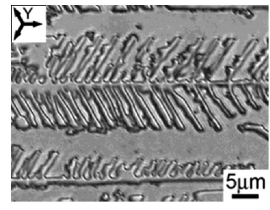

(b)
Fig. 17. (a) Computer simulation of the formation of nanodomain structure in the stripe domain during backswitching. (b) The domain structure appeared as a result of spontaneous backswitching in CLN after external field abrupt switch off. Visualization by optical microscopy.

The formation of nanodomain structures generated under highly nonequilibrium switching conditions arising after applied field abrupt switch off was also investigated by computer simulation within kinetic approach. The process of the spontaneous backswitching in stripe domain was considered. It was shown that the spontaneous backswitching resulted in formation of the connected quasi-regular structure of residual domains (Fig. 17(a)). ${ }^{78}$ This result was confirmed experimentally in LN crystals (Fig. 17(b)). ${ }^{78}$

\section{Nanodomain Engineering}

Since the last decade periodically poled LN (PPLN) is produced industrially for nonlinear optics. The available PPLN with standard 6 to $20 \mu \mathrm{m}$ periods, allows efficient optical parametric interactions using the quasi-phase-matching (QPM) over a wide wavelength range $(0.8-3 \mu \mathrm{m})$, with main applications to tunable coherent sources (optical parametric oscillation, OPO) in the infrared range and all-optical signal processing components working at fiber optics telecommunications wavelengths (wide band amplifiers and frequency converters). ${ }^{28,79,80}$ Nevertheless, those applications are limited to the infrared because of the absence of PPLN with periods below $6 \mu \mathrm{m}$. This bottleneck prevents the usage of PPLN for the realization of OPO working in the visible range.

The interest in short period PPLN goes well beyond its potential usage in standard applications based on co propagative QPM parametric interactions, when all interacting waves: pump, signal, and idler, propagate in the same direction. The realization of PPLN with submicron period would open the counter-propagating optical parametric interactions in which pump and signal or idler waves propagate in opposite directions. This configuration exhibits a large variety of new effects which have been extensively studied theoretically, including realization of a mirror-less OPO ${ }^{81}$ All-optical telecom signal processing, pulse generation using continuous pumping, convective soliton generation and slow light would benefit from this configuration.

The correlated photon sources exhibiting three orders of magnitude narrower bandwidth as compared to the widely used co-propagating sources would have numerous applications in quantum communication. Furthermore, the short period PPLN would allow the realization of electro-optically (EO) switchable Bragg gratings with an unrivalled capacity of integration. Potential applications of those EO Bragg gratings are numerous, in particular for telecom optical components: tunable resonators for tunable fiber or integrated laser sources, gain equalizers for optical amplifiers, optical add-drop multiplexers, and so on. Ferroelectric domain engineering in LN has reached the level where submicron domain structures can be fabricated over a depth of a millimeter; that is, demonstrating aspect ratios exceeding three orders of magnitude.

\section{Conclusion}

The formation of micro- to nanoscale self-assembled domain structures was investigated in single crystals of LN and LT family. The domain structure evolution has been explained within the kinetic approach based on the various nucleation processes similar to the first-order phase transition. The nucleation probability for domain growth is governed by the local value of the electric field averaged over the nucleus size. The used approach to the field induced domain kinetics is based on the consistent accounting of the bulk screening effects and the crucial role of the retardation of residual depolarization field screening. The ineffective screening makes the switching conditions highly nonequilibrium which leads to formation of the self-organized nanodomain structures, such as BDB and web-like nanodomain ensembles due to correlated nucleation, domain growth anisotropy and domain-domain interaction mechanisms. The completely ineffective screening conditions were achieved by acceleration of the domain kinetics or by increasing of the residual depolarization field using various methods: (1) superfast switching, (2) creation of the artificial surface dielectric layer, and (3) switching by pyroelectric field appeared during cooling after pulse heating. The formation of various types of self- 
assembled domain patterns has been revealed at each of five stages of domain structure evolution during polarization reversal. The appearance of oriented nanodomain chains and BDB during polarization reversal with surface layer modified by proton exchange and oriented rays, fractal-type domain structures after pulsed laser irradiation and dendrites during switching at elevated temperatures were revealed at the nucleation stage. The self-assembled dense quasi-regular domain structures were formed in MgOSLT and CLN single crystals during switching in low field applied by solid-state electrodes at the forward growth stage. The growth of selfassembled domain structure with sub-micron fingers was demonstrated at the sideways wall motion stage. The formation of quasi-regular chains of residual domains was revealed in CLN with surface layer modified by proton exchange at the domain merging stage. The correlated nucleation effect has been obtained for the first time in CLN during backswitching stage after the abrupt external field switch-off, the formation of nanodomain rays and quasi-regular structure of fingers was revealed in this case. The obtained quasiregular domain structures can be used for realization of counter-propagating optical parametric interactions and realization of EO switchable Bragg gratings with an unrivalled capacity of integration.

\section{Acknowledgments}

The equipment of the Ural Center for Shared Use "Modern Nanotechnology" Ural Federal University has been used. The research was made possible by Russian Scientific Foundation (Grant 14-12-00826) and in part by Ministry of Education and Science of the Russian Federation (UID RFMEFI59414X0011).

\section{References}

${ }^{1}$ V. Ya. Shur, Handbook of Advanced Dielectric, Piezoelectric and Ferroelectric Materials. Synthesis, Properties and Applications, ed. Z.-G. Ye, Chapter 21, (Woodhead Publishing Ltd, 2008), pp. 622-669.

${ }^{2}$ V. Ya. Shur, Domain nanotechnology in ferroelectrics: Nanodomain engineering in lithium niobate crystals, Ferroelectrics 373, 1 (2008).

${ }^{3}$ V. Ya. Shur, Domain nanotechnology in lithium niobate and lithium tantalate crystals, Ferroelectrics 399, 97 (2010).

${ }^{4}$ V. Ya. Shur, Nucleation Theory and Applications, ed. J.W.P. Schmelzer, Chapter 6 (WILEY-VCH, Weinheim, 2005), pp. 178-214.

${ }^{5} \mathrm{~V}$. Ya. Shur, Kinetics of polarization reversal in normal and relaxor ferroelectrics: relaxation effects, Phase Transit. 65, 49 (1998).

${ }^{6}$ V. Ya. Shur, E. L. Rumyantsev, E. V. Nikolaeva, E. I. Shishkin, D. V. Fursov, R. G. Batchko, L. A. Eyres, M. M. Fejer, R. L. Byer and J. Sindel, Formation of self-organized nanodomain patterns during spontaneous backswitching in lithium niobate, Ferroelectrics 253, 105 (2001).
${ }^{7}$ J. Fousek and L. E. Cross, Open issues in application aspects of domains in ferroic materials, Ferroelectrics 293, 43 (2003).

${ }^{8} \mathrm{~S}$. Wada, Handbook of Advanced Dielectrics Piezoelectric Ferroelectric Materials, ed. Z-G. Ye, Chapter 10 (Woodhead Publishing, 2008), pp. 266-303.

${ }^{9}$ S. Wada, S. Suzuki, T. Noma, T. Suzuki, M. Osada, M. Kakihana, S.-E. Park, L.E. Cross and T. R. Shrout, Enhanced piezoelectric property of barium titanate single crystals with engineered domain configurations, Jpn. J. Appl. Phys. 38, 5505 (1999).

${ }^{10}$ M. Yamada, N. Nada, M. Saitoh and K. Watanabe, Appl. Phys. Lett. 62, 435 (1993).

${ }^{11}$ J. Webjorn, V. Pruneri, P. S. J. Russell, J. R. M. Barr and D. C. Hanna, Electron. Lett. 30, 894 (1994).

${ }^{12}$ L. E. Myers, W. R. Bosenberg, G. D. Miller, R. C. Eckardt, M. M. Fejer and R. L. Byer, Opt. Lett. 20, 52 (1995).

${ }^{13}$ L. E. Myers, R. C. Eckardt, M. M. Fejer, R. L. Byer, W. R. Bosenberg and J. W. Pierce, J. Opt. Soc. Am. B 12, 2102 (1995).

${ }^{14}$ V. Ya. Shur, E. L. Rumyantsev, E. V. Nikolaeva, E. I. Shishkin, D. V. Fursov, R. G. Batchko, L. A. Eyres, M. M. Fejer and R. L. Byer, Nanoscale backswitched domain patterning in lithium niobate, Appl. Phys. Lett. 76, 143 (2000).

${ }^{15}$ V. Ya. Shur, E. Shishkin, E. Rumyantsev, E. Nikolaeva, A. Shur, R. Batchko, M. Fejer, K. Gallo, S. Kurimura, K. Terabe and K. Kitamura, Self-Organization in $\mathrm{LiNbO}_{3}$ and $\mathrm{LiTaO}_{3}$ : formation of micro- and nano-scale domain patterns, Ferroelectrics 304, 111 (2004).

${ }^{16} \mathrm{~V}$. Ya. Shur, Kinetics of ferroelectric domains: Application of general approach to $\mathrm{LiNbO}_{3}$ and $\mathrm{LiTaO}_{3}$, J. Mater. Sci. 41, 199 (2006).

${ }^{17}$ C. Canalias, V. Pasiskevicius, M. Fokine and F. Laurell, Backward quasi-phase-matched second-harmonic generation in submicrometer periodically poled flux-grown $\mathrm{KTiOPO}_{4}$, Appl. Phys. Lett. 86, 181105 (2005).

${ }^{18}$ V. Ya. Shur, Domain nanotechnology in ferroelectric single crystals: lithium niobate and lithium tantalate family, Ferroelectrics 443, 71 (2013).

${ }^{19}$ A. Boes, D. Yudistira, T. Crasto, H. Steigerwald, V. Sivan, T. Limboeck, J. Friend, S. Mailis, E. Soergel and A. Mitchell, Ultraviolet laser induced domain inversion on chromium coated lithium niobate crystals, Opt. Mater. Express 4, 241 (2014).

${ }^{20}$ C. Y. J. Ying, A. C. Muir, C. E. Valdivia, H. Steigerwald, C. L. Sones, R. W. Eason, E. Soergel and S. Mailis, Light-mediated ferroelectric domain engineering and micro-structuring of lithium niobate crystals, Laser Photon. Rev. 6, 526 (2012).

${ }^{21}$ Y. S. Kuz'minov, Lithium Niobate Crystals (Cambridge International Science Publishing, Cambridge, 1997).

${ }^{22}$ K. K. Wong, Properties of Lithium Niobate (The Institution of Engineering and Technology, London, 2002).

${ }^{23} \mathrm{~T}$. Volk and M. Wöhlecke, Lithium Niobate (Springer Berlin Heidelberg, Berlin, Heidelberg, 2008).

${ }^{24}$ V. Ya. Shur, Advanced Piezoelectric Materials: Science and Technology, ed. K. Uchino, Chapter 6 (Woodhead Publishing Ltd, 2010), pp. 204-238.

${ }^{25}$ R. E. Newnham, C. S. Miller, L. E. Cross and T. W. Cline, Tailored domain patterns in piezoelectric crystals, Phys. Status Solidi A 32, 69 (1975).

${ }^{26}$ R. G. Batchko, V. Ya. Shur, M. M. Fejer and R. L. Byer, Backswitch poling in lithium niobate for high-fidelity domain 
patterning and efficient blue light generation, Appl. Phys. Lett. 75, 1673 (1999).

${ }^{27}$ V. Ya. Shur, E. L. Rumyantsev, E. V. Nikolaeva, E. I. Shishkin, R. G. Batchko, G. D. Miller, M. M. Fejer and R. L. Byer, Regular ferroelectric domain array in lithium niobate crystals for nonlinear optic applications, Ferroelectrics 236, 129 (2000).

${ }^{28}$ R. L. Byer, Quasi-phasematched nonlinear interactions and devices, J. Nonlinear Opt. Phys. Mater. 6, 549 (1997).

${ }^{29}$ R. Mitsui, I. Fujii, K. Nakashima, N. Kumada, Y. Kuroiwa and $\mathrm{S}$. Wada, Enhancement in the piezoelectric properties of $\mathrm{BaTiO}_{3}-$ $\mathrm{Bi}\left(\mathrm{Mg}_{1 / 2} \mathrm{Ti}_{1 / 2}\right) \mathrm{O}_{3}-\mathrm{BiFeO}_{3}$ system ceramics by nanodomain, Ceram. Int. 39, S695 (2013).

${ }^{30} \mathrm{E}$. Soergel, Visualization of ferroelectric domains in bulk single crystals, Appl. Phys. B 81, 729 (2005).

${ }^{31}$ V. Ya. Shur and P. S. Zelenovskiy, Micro- and nanodomain imaging in uniaxial ferroelectrics: Joint application of optical, confocal Raman and piezoelectric force microscopy, J. Appl. Phys. 116, 066802 (2014).

${ }^{32}$ V. Ya. Shur, A. I. Lobov, A. G. Shur, S. Kurimura, Y. Nomura, K. Terabe, X. Y. Liu and K. Kitamura, Rearrangement of ferroelectric domain structure induced by chemical etching, Appl. Phys. Lett. 87, 022905 (2005).

${ }^{33}$ C. L. Sones, S. Mailis, W. S. Brocklesby, R. W. Eason and J. R. Owen, Differential etch rates in z-cut $\mathrm{LiNbO}_{3}$ for variable $\mathrm{HF} / \mathrm{HNO}_{3}$ concentrations, J. Mater. Chem. 12, 295 (2002).

${ }^{34} \mathrm{M}$. Alexe and A. L. Gruverman, Nanoscale Characterisation of Ferroelectric Materials: Scanning Probe Microscopy Approach (Springer-Verlag, Berlin, 2004).

${ }^{35}$ S. V. Kalinin and A. L. Gruverman, Scanning Probe Microscopy: Electrical and Electromechanical Phenomena at the Nanoscale (Springer Science+Business Media, LLC, 2007).

${ }^{36}$ A. Gruverman and S. V. Kalinin, Piezoresponse force microscopy and recent advances in nanoscale studies of ferroelectrics, J. Mater. Sci. 41, 107 (2006).

${ }^{37}$ S. V. Kalinin, A. N. Morozovska, L. Q. Chen and B. J. Rodriguez, Local polarization dynamics in ferroelectric materials, Rep. Prog. Phys. 73, 056502 (2010).

${ }^{38}$ S. Kalinin, A. Rar and S. Jesse, A decade of piezoresponse force microscopy: Progress, challenges and opportunities, IEEE Trans. Ultrason. Ferroelectr. Freq. Control 53, 2226 (2006).

${ }^{39} \mathrm{~S}$. V. Kalinin and D. A. Bonnell, Imaging mechanism of piezoresponse force microscopy of ferroelectric surfaces, Phys. Rev. B. 65, 125408 (2002).

${ }^{40}$ V. Ya. Shur, V. A. Shikhova, A. V. Ievlev, P. S. Zelenovskiy, M. M. Neradovskiy, D. V. Pelegov and L. I. Ivleva, Nanodomain structures formation during polarization reversal in uniform electric field in strontium barium niobate single crystals, J. Appl. Phys. 112, 064117 (2012).

${ }^{41}$ V. Ya. Shur, E. I. Shishkin, E. V. Nikolaeva, M. S. Nebogatikov, D. O. Alikin, P. S. Zelenovskiy, M. F. Sarmanova and M. A. Dolbilov, Study of nanoscale domain structure formation using raman confocal microscopy, Ferroelectrics 398, 91 (2010).

${ }^{42}$ V. Ya. Shur, P. S. Zelenovskiy, M. S. Nebogatikov, D. O. Alikin, M. F. Sarmanova, A. V. Ievlev, E. A. Mingaliev and D. K. Kuznetsov, Investigation of the nanodomain structure formation by piezoelectric force microscopy and Raman confocal microscopy in $\mathrm{LiNbO}_{3}$ and $\mathrm{LiTaO}_{3}$ crystals, J. Appl. Phys. 110, 052013 (2011).

${ }^{43}$ E. Fatuzzo and W. J. Merz, Ferroelectricity (North-Holland, Amsterdam, 1967).
${ }^{44}$ R. Miller and G. Weinreich, Mechanism for the sidewise motion of $180^{\circ}$ domain walls in barium titanate, Phys. Rev. 117, 1460 (1960).

${ }^{45}$ V. Ya. Shur, Ferroelectric Thin Films: Synthesis and Basic Properties, Ferroelectricity and Related Phenomena Series, Vol. 10, eds. C. A. Paz de Araujo, J. F. Scott and G. W. Taylor, Chapter 6 (Gordon \& Breach Science Publ., 1996), pp. 153-192.

${ }^{46}$ V. Ya. Shur, A. R. Akhmatkhanov, I. S. Baturin, M. S. Nebogatikov and M. A. Dolbilov, Complex study of bulk screening processes in single crystals of lithium niobate and lithium tantalate family, Phys. Solid State 52, 2147 (2010).

${ }^{47}$ I. S. Baturin, A. R. Akhmatkhanov, V. Ya. Shur, M. S. Nebogatikov, M. A. Dolbilov and E. A. Rodina, Characterization of bulk screening in single crystals of lithium niobate and lithium tantalate family, Ferroelectrics 374, 1 (2008).

${ }^{48}$ V. M. Fridkin, Ferroelectric Semiconductors (Consultants Bureau, New York, 1980).

${ }^{49}$ P. V. Lambeck and G. H. Jonker, The nature of domain stabilization in ferroelectric perovskites, J. Phys. Chem. Solids 47, 453 (1986).

${ }^{50}$ A. K. Tagantsev, I. Stolichnov, E. L. Colla and N. Setter, Polarization fatigue in ferroelectric films: Basic experimental findings, phenomenological scenarios, and microscopic features, J. Appl. Phys. 90, 1387 (2001).

${ }^{51}$ A. K. Tagantsev, L. E. Cross and J. Fousek, Domains in Ferroic Crystals and Thin Films (Springer New York, New York, NY, 2010).

${ }^{52}$ V. Ya. Shur, A. L. Gruverman, N. Yu. Ponomarev and N. A. Tonkachyova, Change of domain structure of lead germanate in strong electric field, Ferroelectrics 126, 371 (1992).

${ }^{53}$ V. Ya. Shur, A. L. Gruverman, N. Yu. Ponomarev, E. L. Rumyantsev and N. A. Tonkachyova, Domain structure kinetics in ultrafast polarization switching in lead germanate, JETP Lett. 53, 615 (1991).

${ }^{54}$ M. A. Dolbilov, E. I. Shishkin, V. Ya. Shur, S. Tascu, P. Baldi and M. P. De Micheli, Abnormal domain growth in lithium niobate with surface layer modified by proton exchange, Ferroelectrics 398, 108 (2010).

${ }^{55}$ M. A. Dolbilov, V. Ya. Shur, E. V. Shishkina, E. S. Angudovich, A. D. Ushakov, P. Baldi and M. P. de Micheli, Formation of nanodomain structure in front of the moving domain wall in lithium niobate single crystal modified by proton exchange, Ferroelectrics 442, 82 (2013).

${ }^{56}$ E. Eliseev, A. Morozovska, G. Svechnikov, E. Rumyantsev, E. Shishkin, V. Shur and S. Kalinin, Screening and retardation effects on $180^{\circ}$-domain wall motion in ferroelectrics: Wall velocity and nonlinear dynamics due to polarization-screening charge interactions, Phys. Rev. B 78, 245409 (2008).

${ }^{57}$ A. Gruverman and A. Kholkin, Nanoscale ferroelectrics: Processing, characterization and future trends, Rep. Prog. Phys. 69, 2443 (2006).

${ }^{58}$ B. J. Rodriguez, R. J. Nemanich, A. Kingon, A. Gruverman, S. V. Kalinin, K. Terabe, X. Y. Liu and K. Kitamura, Domain growth kinetics in lithium niobate single crystals studied by piezoresponse force microscopy, Appl. Phys. Lett. 86, 012906 (2005). ${ }^{59}$ A. V. Ievlev, S. Jesse, A. N. Morozovska, E. Strelcov, E. A. Eliseev, Y. V. Pershin, A. Kumar, V. Ya. Shur and S. V. Kalinin, Intermittency, quasiperiodicity and chaos in probe-induced ferroelectric domain switching, Nat. Phys. 10, 59 (2013). 
${ }^{60}$ R. G. Batchko, V. Ya. Shur, M. M. Fejer and R. L. Byer, Backswitch poling in lithium niobate for high-fidelity domain patterning and efficient blue light generation, Appl. Phys. Lett. 41, 1673 (1999).

${ }^{61}$ V. Shur, E. Rumyantsev, R. Batchko, G. Miller, M. Fejer and R. Byer, Physical basis of the domain engineering in the bulk ferroelectrics, Ferroelectrics 221, 157 (1999).

${ }^{62}$ M. A. Dolbilov, V. Ya. Shur, E. I. Shishkin, M. F. Sarmanova, E. V. Nikolaeva, S. Tascu, P. Baldi and M. P. de Micheli, Influence of surface layers modified by proton exchange on domain kinetics of lithium niobate, Ferroelectrics 374, 14 (2008).

${ }^{63}$ M. P. De Micheli, Fabrication and characterization of proton exchanged waveguides in periodically poled congruent lithium niobate, Ferroelectrics 340, 49 (2006).

${ }^{64}$ E. V. Nikolaeva, V. Ya. Shur, M. A. Dolbilov, E. I. Shishkin, D. K. Kuznetsov, M. F. Sarmanova, O. A. Plaksin and N. V. Gavrilov, Formation of nanoscale domain structures and abnormal switching kinetics in lithium niobate with surface layer modified by implantation of copper ions, Ferroelectrics 374, 73 (2008).

${ }^{65}$ V. Ya. Shur, D. K. Kuznetsov, A. I. Lobov, E. V. Nikolaeva, M. A. Dolbilov, A. N. Orlov and V. V. Osipov, Formation of selfsimilar surface nano-domain structures in lithium niobate under highly nonequilibrium conditions, Ferroelectrics 341, 85 (2006).

${ }^{66}$ D. K. Kuznetsov, V. Ya. Shur, S. A. Negashev, A. I. Lobov, D. V. Pelegov, E. I. Shishkin, P. S. Zelenovskiy, V. V. Platonov, M. G. Ivanov and V. V. Osipov, Formation of nano-scale domain structures in lithium niobate using high-intensity laser irradiation, Ferroelectrics 373, 133 (2008).

${ }^{67}$ V. Ya. Shur, D. K. Kuznetsov, A. I. Lobov, D. V. Pelegov, E. V. Pelegova, V. V. Osipov, M. G. Ivanov and A. N. Orlov, Selfsimilar surface nanodomain structures induced by laser irradiation in lithium niobate, Phys. Solid State 50, 717 (2008).

${ }^{68}$ D. K. Kuznetsov, V. Ya. Shur, E. A. Mingaliev, S. A. Negashev, A. I. Lobov, E. L. Rumyantsev and P. A. Novikov, Nanoscale domain structuring in lithium niobate single crystals by pulse laser heating, Ferroelectrics 398, 49 (2010).

${ }^{69}$ E. A. Mingaliev, V. Ya. Shur, D. K. Kuznetsov, S. A. Negashev and A. I. Lobov, Formation of stripe domain structures by pulse laser irradiation of $\mathrm{LiNbO}_{3}$ crystals, Ferroelectrics 399, 7 (2010).
${ }^{70}$ A. I. Lobov, V. Ya. Shur, D. K. Kuznetsov, S. A. Negashev, D. V. Pelegov, E. I. Shishkin and P. S. Zelenovskiy, Discrete switching by growth of nano-scale domain rays under highlynonequilibrium conditions in lithium niobate single crystals, Ferroelectrics 373, 99 (2008).

${ }^{71}$ A. R. Akhmatkhanov, V. Ya. Shur, I. S. Baturin, D. V. Zorikhin, A. M. Lukmanova, P. S. Zelenovskiy and M. M. Neradovskiy, Domain kinetics in lithium niobate single crystals with photoresist dielectric layer, Ferroelectrics 439, 3 (2012).

${ }^{72}$ J. Feder, Fractals (Plenum, New York, 1988).

${ }^{73}$ V. Ya. Shur, D. S. Chezganov, M. S. Nebogatikov, I. S. Baturin and M. M. Neradovskiy, Formation of dendrite domain structures in stoichiometric lithium niobate at elevated temperatures, J. Appl. Phys. 112, 104113 (2012).

${ }^{74}$ A. R. Akhmatkhanov, M. A. Chuvakova, I. S. Baturin and V. Ya. Shur, Formation of self-assembled domain structures in MgOSLT, Ferroelectrics 476, 76 (2015).

${ }^{75}$ V. Ya. Shur, A. R. Akhmatkhanov and I. S. Baturin, Fatigue effect in ferroelectric crystals: Growth of the frozen domains, J. Appl. Phys. 111, 124111 (2012).

${ }^{76}$ V. Ya. Shur, A. R. Akhmatkhanov and I. S. Baturin, Fatigue effect in stoichiometric $\mathrm{LiTaO}_{3}$ crystals produced by vapor transport equilibration, Ferroelectrics 426, 142 (2012).

${ }^{77}$ V. Ya. Shur, E. L. Rumyantsev, E. V. Nikolaeva and E. I. Shishkin, Formation and evolution of charged domain walls in congruent lithium niobate, Appl. Phys. Lett. 77, 3636 (2000).

${ }^{78}$ V. Ya. Shur, A. I. Lobov, E. L. Rumyantsev and D. K. Kuznetsov, 3D modeling of domain structure evolution during discrete switching in lithium niobate, Ferroelectrics 399, 68 (2010).

${ }^{79}$ J. Armstrong, N. Bloembergen, J. Ducuing and P. Pershan, Interactions between light waves in a nonlinear dielectric, Phys. Rev. 127, 1918 (1962)

${ }^{80}$ D. S. Hum and M. M. Fejer, Quasi-phasematching, C. R. Phys. 8, 180 (2007).

${ }^{81}$ S. E. Harris, Proposed backward wave oscillation in the infrared, Appl. Phys. Lett. 9, 114 (1966). 